\title{
Hyperscaling violation, quasinormal modes and shear diffusion
}

\section{Debangshu Mukherjee and Krishnan Narayan}

Chennai Mathematical Institute, SIPCOT IT Park, Siruseri 603103, India

E-mail: debangshu@cmi.ac.in, narayan@cmi.ac.in

ABSTRACT: We study quasinormal modes of shear gravitational perturbations for hyperscaling violating Lifshitz theories, with Lifshitz and hyperscaling violating exponents $z$ and $\theta$. The lowest quasinormal mode frequency yields a shear diffusion constant which is in agreement with that obtained in previous work by other methods. In particular for theories with $z<d_{i}+2-\theta$ where $d_{i}$ is the boundary spatial dimension, the shear diffusion constant exhibits power-law scaling with temperature, while for $z=d_{i}+2-\theta$, it exhibits logarithmic scaling. We then calculate certain 2-point functions of the dual energy-momentum tensor holographically for $z \leq d_{i}+2-\theta$, identifying the diffusive poles with the quasinormal modes above. This reveals universal behaviour $\eta / s=1 / 4 \pi$ for the viscosity-to-entropy-density ratio for all $z \leq d_{i}+2-\theta$.

KEYWORDS: Gauge-gravity correspondence, Holography and condensed matter physics (AdS/CMT)

ARXIV EPRINT: 1707.07490 


\section{Contents}

1 Introduction 1

2 Hyperscaling violating Lifshitz (hvLif) theory 2

2.1 hvLif in absence of gauge field: dilaton gravity, $z=1, d_{i}=2 \quad 4$

2.2 hvLif theory: generalized analysis 6

$\begin{array}{lll}\text { 2.2.1 Solving for gauge field perturbation } a_{y} & 9\end{array}$

3 Dual field theory correlation functions $\quad 10$

3.1 Dual field theory correlation functions: $z=d_{i}+2-\theta \quad 11$

4 Discussion $\quad 12$

$\begin{array}{ll}\text { A Reviewing hyperscaling violating Lifshitz spacetimes } & 13\end{array}$

B Solution for gauge field perturbation $a_{y}$ : details $\quad 14$

$\begin{array}{ll}\text { C Shear diffusion and } \frac{\eta}{s} \text { from membrane paradigm } & 16\end{array}$

\section{Introduction}

Over the last several years, the framework of gauge/gravity duality [1-4] has been generalized and applied to understand strongly coupled non-relativistic field theories: see e.g. [5] for a recent review. A class of these, dubbed hyperscaling violating Lifshitz (hvLif) theories, has been studied extensively. These are conformally Lifshitz solutions to effective Einstein-Maxwell-Dilaton theories. Some of these exhibit novel entanglement scaling [6-8], reflected also in certain string realizations [9-13].

It is of great interest to understand these nonrelativistic theories with regard to their low energy behaviour, in particular hydrodynamics and the viscosity bound [14]. Some previous investigations appear in e.g. [15-27], in part reviewed in [5]. In the context of gauge/gravity duality [1-4] for relativistic theories, various transport properties are encoded in the quasinormal modes of the dual gravitational black branes, see e.g. [28-31]. Quasinormal modes are solutions to the linearized equations governing the gravitational perturbations that are ingoing at the horizon and vanishing at the boundary: these boundary conditions make the low lying hydrodynamic modes damped and diffusive, with a dispersion relation that encodes the hydrodynamic diffusive poles in certain 2-point correlation functions in the dual field theory. 
Motivated by these earlier studies, in this paper we analyse the lowest quasinormal mode spectrum for shear gravitational perturbations in hyperscaling violating Lifshitz theories with Lifshitz exponent $z$ and hyperscaling violating exponent $\theta$. We turn on appropriate metric and gauge field perturbations $h_{x y}, h_{t y}$ and $a_{y}$ of the form $e^{-i \omega t+i q x}$. Defining appropriate new field variables $\mathcal{H}$ invariant under a residual gauge symmetry for such perturbations enables us to identify the relevant differential equations governing these modes. The hydrodynamic regime allows the approximation of low frequency and momentum relative to the temperature scale. Then using $\boldsymbol{\Omega} \sim \frac{\omega}{T} \ll 1$ and $\mathbf{Q} \sim \frac{q}{T^{1 / z}} \ll 1$, as expansion parameters, we find series solutions for the quasinormal modes. The lowest quasinormal modes for these shear perturbations are of the form $\omega=-i \mathcal{D} q^{2}$ where $\mathcal{D}$ is the shear diffusion constant. Our analysis (section 2) of these quasinormal modes and the associated boundary conditions can be carried out provided the exponents satisfy $z \leq d_{i}+2-\theta$. In particular $\mathcal{D}$ exhibits power law scaling with temperature for $z<d_{i}+2-\theta$. The shear diffusion constant $\mathcal{D}$ for hvLif theories obtained thus is in agreement with that obtained previously in $[25,27]$, where $\mathcal{D}$ was obtained by adapting the "membrane paradigm" approach of [32]. To elaborate further, turning on perturbations $h_{x y}, h_{t y}$ and $a_{y}$ and further compactifying the theory along a spatial direction exhibiting translation invariance, we mapped near-horizon metric perturbations to gauge field perturbations in an auxilliary theory in one lower dimension. The gauge fields were used to define currents $j^{\mu}$ on a "stretched horizon", satisfying a diffusion equation $\partial_{t} j^{t}=\mathcal{D} \partial_{x}^{2} j^{t}$. The shear diffusion constant $\mathcal{D}$ is obtained by solving for the perturbations using a set of self-consistent assumptions in a near horizon expansion. This membrane paradigm approach does not require holography as such.

In section 3 using the asymptotic behaviour of the quasinormal mode perturbations in section 2 above, we adapt the prescription of [33, 34] to compute certain 2-point correlation functions of the dual energy-momentum tensor operators. The poles of these retarded correlators are identical to the lowest quasinormal frequencies above of the dual black brane for $z<d_{i}+2-\theta$, vindicating the correspondence between quasinormal mode frequencies, the shear diffusion constant and the poles of the retarded correlators for nonrelativistic theories with $z<d_{i}+2-\theta$, thereby giving $\frac{\eta}{s}=\frac{1}{4 \pi}$.

For $z=d_{i}+2-\theta$, the shear diffusion constant above exhibits logarithmic scaling, the logarithm containing the ultraviolet cutoff. However the correlation functions obtained above, in the Kubo limit, continue to reveal universal behaviour for the viscosity bound

with $\frac{\eta}{s}=\frac{1}{4 \pi}$ as we discuss in section 3.1. In appendix A and B, we provide some technical details, and in appendix $\mathrm{C}$, we review the membrane paradigm approach to the shear diffusion constant studied in $[25,27]$.

\section{Hyperscaling violating Lifshitz (hvLif) theory}

In this section, we describe the nonrelativistic holographic backgrounds in which we want to study quasinormal mode solutions. These backgrounds of interest here are described by 
$(d+1)$-dimensional hyperscaling violating metrics at finite temperature given by

$$
d s^{2}=r^{2 \theta / d_{i}}\left(-\frac{f(r)}{r^{2 z}} d t^{2}+\frac{d r^{2}}{f(r) r^{2}}+\sum_{d_{i}} \frac{d x_{i}^{2}}{r^{2}}\right), \quad d_{i}=d-1, \quad f(r)=1-\left(r_{0} r\right)^{d_{i}+z-\theta} .
$$

$r=\frac{1}{r_{0}}$ is the location of the horizon, and $d_{i}$ is the boundary spatial dimension. These are conformally Lifshitz solutions to Einstein-Maxwell-dilaton theories (see appendix A for some details). The temperature of the field theory dual to the hvLif theory (2.1) is the Hawking temperature of the black brane

$$
T=\frac{d_{i}+z-\theta}{4 \pi} r_{0}^{z}
$$

We are interested in studying shear gravitational modes: these are the modes $h_{x y}$ and $h_{t y}$, which in general couple to the gauge field perturbations $a_{y}$. We turn on perturbations of the form $e^{-i \omega t+i q x} h_{\mu \nu}(r), e^{-i \omega t+i q x} a_{\mu}(r)$, and restrict ourselves to radial gauge $\left(h_{\mu r}=\right.$ $\left.a_{r}=0\right)$. Then, as in [25, 27], shear diffusion can be studied by mapping it to charge diffusion in a theory in one lower dimension obtained by compactifying one of the spatial directions $x_{i}$ enjoying translation invariance, say $y$. Motivated by this, we define the variables

$$
H_{t y}=g^{x x} h_{t y}=r^{2-\frac{2 \theta}{d_{i}}} h_{t y}, \quad H_{x y}=g^{x x} h_{x y}=r^{2-\frac{2 \theta}{d_{i}}} h_{x y} .
$$

Then the equations of motion governing the perturbations are simply

$$
\begin{aligned}
\partial_{r}\left(r^{z+\theta-\left(d_{i}+1\right)} H_{t y}^{\prime}\right)-k a_{y}^{\prime}-\frac{r^{z+\theta-\left(d_{i}+1\right)}}{f} q\left(\omega H_{x y}+q H_{t y}\right) & =0, \\
\partial_{r}\left(r^{\theta-z-d_{i}+1} f H_{x y}^{\prime}\right)+\frac{r^{z+\theta-\left(d_{i}+1\right)}}{f} \omega\left(\omega H_{x y}+q H_{t y}\right) & =0, \\
q r^{2-2 z} H_{x y}^{\prime}+\frac{\omega}{f}\left(H_{t y}^{\prime}-k r^{\left(d_{i}+1\right)-z-\theta} a_{y}\right) & =0, \\
\partial_{r}\left(r^{d_{i}+3-z-\theta} f a_{y}^{\prime}\right)+\frac{r^{d_{i}+1+z-\theta}}{f} \omega^{2} a_{y}-r^{d_{i}+3-z-\theta} q^{2} a_{y}-k H_{t y}^{\prime} & =0,
\end{aligned}
$$

where $k=\left(d_{i}+z-\theta\right) \alpha$ and $\alpha=-\sqrt{\frac{2(z-1)}{d_{i}+z-\theta}}$ (see (A.3)). The first three equations, namely (2.4), (2.5) and (2.6) are the three relevant components of the Einstein equations while (2.7) is the linearized Maxwell's equation: we refer to [27] for details (in part reviewed in the appendix). Now, following [28, 29], we note that there is a residual gauge invariance in these variables representing fluctuations of the form above: the metric fluctuations transform under infinitesimal diffeomorphisms as $h_{\mu \nu} \rightarrow h_{\mu \nu}-\nabla_{\mu} \xi_{\nu}-\nabla_{\nu} \xi_{\mu}$, with the gauge functions $\xi_{\mu}(t, x, r) \equiv \xi_{\mu}(r) e^{-i \omega t+i q x}$. The residual gauge invariance then allows us to consider the following gauge invariant combination defined as

$$
\mathcal{H}=\omega H_{x y}+q H_{t y}-k q \int_{r_{c}}^{r} s^{d_{i}+1-z-\theta} a_{y}(s) d s .
$$

Note that the gauge field component $a_{y}$ is invariant by itself. This combination is motivated by the investigation in [27] where a similar combination (C.1) appears as the field variable 
(mixing $h_{t y}, a_{y}$ perturbations) that allows a realization of the diffusion equation from the Einstein equations governing the near horizon shear perturbations: this is reviewed in appendix C. Under a compactification of the $y$-direction, these metric components become gauge field components with a residual $\mathrm{U}(1)$ gauge invariance. We will see the role of this variable $\mathcal{H}$ in what follows. The equations of motion i.e. (2.4)-(2.7) can be finally reduced to a system of two coupled second order equations using the field $\mathcal{H}$ defined above in (2.8), i.e. (primes denote $r$-derivatives)

$$
\begin{gathered}
\mathcal{H}^{\prime \prime}+\left[\partial_{r} \log r^{z+\theta-\left(d_{i}+1\right)}+\frac{\mathbf{\Omega}^{2}}{\mathbf{\Omega}^{2}-(2 \pi T)^{2 / z-2} \mathbf{Q}^{2} f r^{2-2 z}} \partial_{r} \log \left(r^{2-2 z} f\right)\right] \mathcal{H}^{\prime} \\
+(2 \pi T)^{2}\left(\frac{r^{2 z-2}}{f^{2}} \mathbf{\Omega}^{2}-(2 \pi T)^{2 / z-2} \frac{\mathbf{Q}^{2}}{f}\right) \mathcal{H} \\
+(2 \pi T)^{2+1 / z} k \mathbf{Q}\left(\frac{r^{2 z-2}}{f^{2}} \mathbf{\Omega}^{2}-(2 \pi T)^{2 / z-2} \frac{\mathbf{Q}^{2}}{f}\right) \int_{0}^{r} d s s^{\left(d_{i}+1\right)-z-\theta} a_{y}=0, \\
a_{y}^{\prime \prime}+\left[\partial_{r} \log f r^{d_{i}+3-z-\theta}\right] a_{y}^{\prime}+\left((2 \pi T)^{2} \frac{r^{2 z-2}}{f^{2}} \mathbf{\Omega}^{2}-(2 \pi T)^{2 / z} \frac{\mathbf{Q}^{2}}{f}-\frac{k^{2}}{r^{2} f}\right) a_{y} \\
+\frac{(2 \pi T)^{1 / z-2} k \mathbf{Q} \cdot r^{\theta-z-d_{i}-1}}{\mathbf{\Omega}^{2}-(2 \pi T)^{2 / z-2} \mathbf{Q}^{2} f r^{2-2 z}} \mathcal{H}^{\prime}=0 .
\end{gathered}
$$

Here, $\boldsymbol{\Omega}$ and $\mathbf{Q}$ satisfying

$$
\mathbf{\Omega}=\frac{\omega}{2 \pi T}, \quad \mathbf{Q}=\frac{q}{(2 \pi T)^{1 / z}}, \quad \mathbf{\Omega}, \mathbf{Q} \ll 1,
$$

are combinations that are dimensionless for the $z=1$ AdS case, and Lifshitz invariant for $\theta=0$. The condition $\boldsymbol{\Omega}, \mathbf{Q} \ll 1$ in (2.11) is imposed to restrict to the hydrodynamic regime where we can identify appropriate quasinormal mode solutions and frequencies to the above equations. These are solutions to the above differential equations governing the perturbations which are ingoing at the horizon and vanishing at the boundary (far from the horizon): they are damped modes reflecting diffusion in these backgrounds and describe how the perturbed system "settles down". In relativistic theories, quasinormal modes are known to be closely related to hydrodynamic diffusive modes and the associated diffusive poles in the dual field theories. In what follows, we will generalize these studies to hvLif theories. Restricting to the hydrodynamic regime enables us to look for solutions to (2.9) and (2.10) in a perturbative series. It turns out that the leading and next-to-leading behaviour for the mode $\mathcal{H}$ can be determined independent of the $a_{y}$ solution (which can then be solved for using the solution of $\mathcal{H}$ ).

\section{1 hvLif in absence of gauge field: dilaton gravity, $z=1, d_{i}=2$}

In this subsection, we will analyse a simple case of dilaton gravity in 4 bulk dimensions $\left(d_{i}=2\right)$ as a warmup example. It can be easily seen from (A.1), that in the absence of a background gauge field $\left(A_{\mu}=0\right)$, the hvLif theory reduces to a theory of a scalar field (dilaton) coupled to gravity with $z=1$ and $k=0$ (which follows from (A.3)). The gauge invariant combination $\mathcal{H}$ defined in (2.8) takes a simpler form and satisfies

$$
\mathcal{H}=\omega H_{x y}+q H_{t y}: \quad \mathcal{H}^{\prime \prime}-\frac{P^{\prime}}{P} \mathcal{H}^{\prime}+(2 \pi T)^{2}\left(\frac{\mathbf{\Omega}^{2}}{f^{2}}-\frac{\mathbf{Q}^{2}}{f}\right) \mathcal{H}=0 .
$$


simplifying (2.9), and we have defined the function $P(r) \equiv \frac{\mathbf{\Omega}^{2}-\mathbf{Q}^{2} f(r)}{f(r) r^{\theta-2}}$. Close to the horizon (as $r \rightarrow \frac{1}{r_{0}}$ ), the above equation can be approximated as

$$
\mathcal{H}^{\prime \prime}+\frac{f^{\prime}}{f} \mathcal{H}^{\prime}+\frac{(2 \pi T)^{2} \boldsymbol{\Omega}^{2}}{f^{2}} \mathcal{H}=0
$$

Using an asymptotic scaling ansatz of the form $\mathcal{H} \sim f^{A}$ in this equation yields $A= \pm \frac{i \boldsymbol{\Omega}}{2}$. Choosing the exponent $A=-\frac{i \Omega}{2}$, and restoring the explicit time-dependence, we see that $\mathcal{H} \sim e^{-i \omega\left(t+\frac{1}{4 \pi T} \log f(r)\right)}$. With $z=1, d_{i}=2$, from $(2.1)$ we have $f(r)=1-\left(r_{0} r\right)^{3-\theta}$ with the boundary defined at $r \rightarrow 0$. The blackening factor turns off as $f(r) \rightarrow 1$ far from the horizon only if $\theta<3$. Focussing therefore on $\theta<3$ from now on, we see that as time evolves (increasing $t$ ), these modes carry energy towards the horizon, i.e. these are ingoing modes at the horizon. Taking the ansatz

$$
\mathcal{H}(r, \boldsymbol{\Omega}, \mathbf{Q})=f(r)^{-\frac{i \Omega}{2}} F(r, \boldsymbol{\Omega}, \mathbf{Q}),
$$

and using in (2.12), we can obtain a second order equation governing $F(r, \boldsymbol{\Omega}, \mathbf{Q})$. Towards studying hydrodynamic modes, we analyse (2.12) in the regime $\boldsymbol{\Omega} \ll 1, \mathbf{Q} \ll 1$. To keep track of the order of the perturbative solution, we introduce a book-keeping parameter $\lambda$ and rescale $\boldsymbol{\Omega} \rightarrow \lambda \boldsymbol{\Omega}$ and $\mathbf{Q} \rightarrow \lambda \mathbf{Q}$, following [28, 29]. Rescaling then gives

$$
\begin{aligned}
F^{\prime \prime}-\left(i \lambda \Omega \frac{f^{\prime}}{f}+\frac{P^{\prime}}{P}\right) F^{\prime}+\left(-\frac{i \lambda \Omega}{2}\left(\frac{f^{\prime}}{f}\right)^{\prime}\right. & \left.-\frac{\lambda^{2} \boldsymbol{\Omega}^{2}}{4}\left(\frac{f^{\prime}}{f}\right)^{2}+\frac{i \lambda \Omega}{2} \frac{f^{\prime}}{f} \frac{P^{\prime}}{P}\right) F \\
& +(2 \pi T)^{2} \lambda^{2}\left(\frac{\Omega^{2}}{f^{2}}-\frac{\mathbf{Q}^{2}}{f}\right) F=0 .
\end{aligned}
$$

Assuming that the solution admits a series expansion in the perturbation parameter $\lambda$ i.e.

$$
F(r)=F_{0}(r)+\lambda F_{1}(r)+\lambda^{2} F_{2}(r)+\cdots,
$$

we can write a second order equation for $F_{0}(r)$ and its corresponding solution as

$$
F_{0}^{\prime \prime}-\frac{P^{\prime}}{P} F_{0}^{\prime}=0 ; \quad F_{0}(r)=C_{0}+C_{1} \int^{r} \frac{\Omega^{2}-\mathbf{Q}^{2} f}{f r^{\prime-2}} \cdot d r^{\prime} \stackrel{\text { regularity }}{\longrightarrow} \quad F_{0}(r)=C_{0} .
$$

Near the horizon, $f(r)$ vanishes, giving a logarithmic divergence in $F_{0}$. Demanding regularity of the solution at the horizon forces us to set $C_{1}=0$, thus giving the solution as simply $F_{0}=C_{0}$ in (2.17). Using this in (2.15) and collecting terms of $O(\lambda)$ gives an inhomogeneous differential equation for $F_{1}(r)$,

$$
F_{1}^{\prime \prime}-\frac{P^{\prime}}{P} F_{1}^{\prime}=C_{0} \frac{i \Omega}{2}\left[\left(\frac{f^{\prime}}{f}\right)^{\prime}-\frac{P^{\prime}}{P} \cdot \frac{f^{\prime}}{f}\right] .
$$

Integrating and multiplying throughout by $P$, we get

$$
F_{1}^{\prime}=\frac{i \boldsymbol{\Omega}}{2} C_{0} \partial_{r} \log f+\kappa_{1} P \quad \Rightarrow \quad \kappa_{1}=-\frac{i C_{0}}{2 \boldsymbol{\Omega}} f^{\prime}\left(1 / r_{0}\right) r_{0}^{2-\theta}=\frac{i C_{0}}{2 \boldsymbol{\Omega}}(3-\theta) r_{0}^{3-\theta} .
$$


The above value for the constant $\kappa_{1}$ is required by demanding regularity of $F_{1}$ at the horizon which implies $F_{1}^{\prime}$ must be finite as $r \rightarrow \frac{1}{r_{0}}$. Using this value of $\kappa_{1}$, the solution to $(2.18)$ is

$$
F_{1}(r)-F_{1}\left(1 / r_{0}\right)=\frac{i C_{0} \mathbf{Q}^{2}}{2 \boldsymbol{\Omega}}\left(1-\left(r_{0} r\right)^{3-\theta}\right) .
$$

We set the integration constant $F_{1}\left(1 / r_{0}\right)$ to zero, as in [34]. This is consistent with the absence of any additional dependence on $\boldsymbol{\Omega}, \mathbf{Q}$ in the subleading terms $F_{i}(r)$ in (2.16), i.e. with fixing the normalization of the modes as simply $C_{0} e^{-i \omega t} f(r)^{-i \Omega / 2}$ at the horizon.

Imposing the Dirichlet boundary condition $\mathcal{H}(0)=0$, i.e. the fluctuations vanish on the boundary $r=r_{c} \rightarrow 0$, we obtain

$$
1+\left.\frac{i \mathbf{Q}^{2}}{2 \boldsymbol{\Omega}}\left(1-\left(r_{0} r\right)^{3-\theta}\right)\right|_{r \sim 0}=0 .
$$

Using (2.11), at the boundary, we thus obtain the dispersion relation

$$
\omega=-i \frac{1}{4 \pi T} q^{2} \equiv-i \mathcal{D} q^{2}
$$

where $\mathcal{D}=\frac{1}{4 \pi T}$ is the shear diffusion constant. This is consistent with $\mathcal{D}$ found in $[25$, 27], using a membrane-paradigm-like near horizon analysis (generalizing [32]), and the corresponding guess $\frac{\eta}{s}=\mathcal{D} T=\frac{1}{4 \pi}$ for universal viscosity-to-entropy-density.

\section{2 hvLif theory: generalized analysis}

In this section, we will study hvLif theories in full generality. To study hydrodynamics, we will focus on the regime $\Omega \ll 1, \mathbf{Q} \ll 1$, taking the ansatze

$$
\mathcal{H}(r, \boldsymbol{\Omega}, \mathbf{Q})=f^{\frac{-i \boldsymbol{\Omega}}{2}} F(r, \boldsymbol{\Omega}, \mathbf{Q}) ; \quad a_{y}(r, \boldsymbol{\Omega}, \mathbf{Q})=f^{\frac{-i \boldsymbol{\Omega}}{2}} G(r, \boldsymbol{\Omega}, \mathbf{Q}) .
$$

The factor $f^{-\frac{i \Omega}{2}}$ reflects the "ingoing" nature of these solutions, as in the previous $z=1$ case. The null energy condition (A.5) implies $d_{i}+z-\theta>0$ for theories with $z>1$ so the factor $f^{-\frac{i \Omega}{2}}$ always reflects "infalling" modes noting the form of $f(r)$ in (2.1). Rewriting (2.9) in terms of $F$ and $G$ and further rescaling $\boldsymbol{\Omega} \rightarrow \lambda \boldsymbol{\Omega}, \mathbf{Q} \rightarrow \lambda \mathbf{Q}$, we end up with

$$
\begin{aligned}
& F^{\prime \prime}-\left(\frac{H^{\prime}}{H}+i \lambda \boldsymbol{\Omega} \frac{f^{\prime}}{f}\right) F^{\prime} \\
& -\left[\frac{i \lambda \boldsymbol{\Omega}}{2}\left(\frac{f^{\prime}}{f}\right)^{\prime}+\frac{\lambda^{2} \boldsymbol{\Omega}^{2}}{4}\left(\frac{f^{\prime}}{f}\right)^{2}-\frac{i \lambda \boldsymbol{\Omega}}{2} \frac{f^{\prime} H^{\prime}}{f H}-\lambda^{2}(2 \pi T)^{2} \frac{r^{2 z-2}}{f^{2}}\left(\boldsymbol{\Omega}^{2}-(2 \pi T)^{\frac{2}{z}-2} \mathbf{Q}^{2} f r^{2-2 z}\right)\right] F \\
& +\lambda^{3}(2 \pi T)^{2+\frac{1}{z}} k \mathbf{Q} \frac{r^{2 z-2}}{f^{2}}\left(\boldsymbol{\Omega}^{2}-(2 \pi T)^{\frac{2}{z}-2} \mathbf{Q}^{2} f r^{2-2 z}\right) f^{\frac{i \lambda \boldsymbol{\Omega}}{2}} \int d s \cdot f^{-\frac{i \lambda \boldsymbol{\Omega}}{2}} s^{d_{i}+1-z-\theta} G=0 .
\end{aligned}
$$

We also assume the solutions admit a series expansion in $\lambda$ as following

$$
\begin{aligned}
& F(r, \boldsymbol{\Omega}, \mathbf{Q})=F_{0}(r, \boldsymbol{\Omega}, \mathbf{Q})+\lambda F_{1}(r, \boldsymbol{\Omega}, \mathbf{Q})+O\left(\lambda^{2}\right)+\cdots \\
& G(r, \boldsymbol{\Omega}, \mathbf{Q})=G_{0}(r, \boldsymbol{\Omega}, \mathbf{Q})+\lambda G_{1}(r, \boldsymbol{\Omega}, \mathbf{Q})+O\left(\lambda^{2}\right)+\cdots
\end{aligned}
$$

Gathering terms order-by-order, we see that $F_{0}$ follows a homogeneous second order differential equation while the nature of $F_{1}$ depends on $F_{0}$. We have argued in appendix B 
that the last term in (2.24) becomes relevant only at $O\left(\lambda^{3}\right)$ and does not contribute to the $F_{0}$ and $F_{1}$ solutions. In section 2.2.1, we demonstrate that the $F_{0}$ and $F_{1}$ solutions are consistent with (2.10) too and solve for the function $G_{0}(r, \boldsymbol{\Omega}, \mathbf{Q})$ which is determined by $F_{0}$ and $F_{1}$. Further $G_{1}$ requires knowledge of $F_{2}$ also. Thus although the exact form of the perturbation solutions $\mathcal{H}, a_{y}$, is governed by the coupled equations (2.9), (2.10), restricting to $O(\lambda)$ essentially decouples the $a_{y}$ terms from the equation governing $\mathcal{H}$ which we will solve for below.

Sticking (2.25) in (2.24) and gathering terms of $O\left(\lambda^{0}\right)$ gives

$$
F_{0}^{\prime \prime}-\frac{H^{\prime}}{H} F_{0}^{\prime}=0 \quad \text { with } \quad H=\frac{\Omega^{2}-(2 \pi T)^{2 / z-2} \mathbf{Q}^{2} f r^{\prime 2-2 z}}{f r^{\prime \theta-z-d_{i}+1}} \stackrel{\text { regularity }}{\longrightarrow} F_{0}=C_{0} .
$$

To elaborate, the solution to the equation above (analogous to (2.17)) is $F_{0}=C_{0}+C_{1} \int H d r$ and regularity of $F_{0}$ requires $C_{1}=0$ giving $F_{0}=C_{0}$ above. The next-to-leading solution $F_{1}$ satisfies an equation structurally similar to (2.18),

$$
F_{1}^{\prime \prime}-\frac{H^{\prime}}{H} F_{1}^{\prime}=C_{0} \frac{i \Omega}{2}\left[\left(\frac{f^{\prime}}{f}\right)^{\prime}-\frac{H^{\prime}}{H} \cdot \frac{f^{\prime}}{f}\right] .
$$

Integrating gives

$$
F_{1}^{\prime}=\frac{i \boldsymbol{\Omega} C_{0}}{2} \partial_{r} \log f+\kappa_{2} \cdot \frac{\boldsymbol{\Omega}^{2}-(2 \pi T)^{2 / z-2} \mathbf{Q}^{2} f r^{2-2 z}}{f r^{\theta-z-d_{i}+1}} \Rightarrow \kappa_{2}=\frac{i C_{0}}{2 \boldsymbol{\Omega}}\left(d_{i}+z-\theta\right) r_{0}^{d_{i}+z-\theta} .
$$

The integration constant $\kappa_{2}$ is fixed as in (2.19) by demanding regularity of $F_{1}$ at the horizon $r \rightarrow 1 / r_{0}$. This forces the singular part from the first term to be cancelled by the other singular piece coming from the $O\left(\boldsymbol{\Omega}^{2}\right)$ term, fixing $\kappa_{2}$ above. The value of $\kappa_{2}$ can be used to write down the solution to $F_{1}(r)$,

$$
F_{1}(r)=-\frac{i C_{0}\left(d_{i}+z-\theta\right) \mathbf{Q}^{2}}{2 \boldsymbol{\Omega}}(2 \pi T)^{\frac{2}{z}-2} r_{0}^{d_{i}+z-\theta} \int_{\frac{1}{r_{0}}}^{r} r^{\prime d_{i}+1-z-\theta} d r^{\prime} .
$$

As in (2.20) and the comments following it, we have set the integration constant $F_{1}\left(1 / r_{0}\right)$ to zero in the second line. Then the solution to (2.9) upto first order in the hydrodynamic expansion can be written down and varies depending on the value of $\left(d_{i}, z, \theta\right)$.

$\boldsymbol{z}<\boldsymbol{d}_{\boldsymbol{i}}+\mathbf{2}-\boldsymbol{\theta}$. This is the sector continuously connected to relativistic (AdS) theories which have $z=1, \theta=0$. This sector also includes hvLif theories arising from reductions of $p \leq 4$ nonconformal $D p$-branes where $z=1, d_{i}=p, \theta=p-\frac{9-p}{5-p}$. The solution to (2.9) upto first order is given by

$$
\mathcal{H}=C_{0} f(r)^{-\frac{i \Omega}{2}}\left[1+\frac{i q^{2}}{\left(d_{i}+2-z-\theta\right) \omega} r_{0}^{z-2} \cdot\left(1-\left(r_{0} r\right)^{d_{i}+2-z-\theta}\right)\right]
$$

where $r_{0}$ is related to the temperature $T$ as in (2.2). Imposing Dirichlet boundary conditions i.e. $\mathcal{H}(r \rightarrow 0)=0$ at the UV cut-off boundary $\left(r=r_{c} \rightarrow 0\right)$ using $(2.11)$ gives

$$
\omega=-i q^{2} \cdot \frac{1}{d_{i}+2-z-\theta} \cdot\left(\frac{4 \pi T}{d_{i}+z-\theta}\right)^{1-2 / z} \equiv-i \mathcal{D} q^{2}
$$


as the quasinormal mode frequency. This gives the leading shear diffusion constant

$$
\mathcal{D}=\frac{r_{0}^{z-2}}{d_{i}+2-z-\theta}=\frac{1}{d_{i}+2-z-\theta} \cdot\left(\frac{4 \pi}{d_{i}+z-\theta}\right)^{1-2 / z} T^{\frac{z-2}{z}},
$$

which matches the result obtained using the membrane paradigm approach in $[25,27]$ (reviewed in appendix C). This led to a guess for the relation between the shear diffusion constant $\mathcal{D}$ and shear viscosity $\eta$, consistent with various special cases,

$$
\frac{\eta}{s}=\frac{d_{i}+2-z-\theta}{4 \pi}\left(\frac{4 \pi}{d_{i}+z-\theta}\right)^{\frac{2-z}{z}} \mathcal{D} T^{\frac{2-z}{z}}=\frac{1}{4 \pi} .
$$

We will later (section 3) evaluate the viscosity using holographic techniques, corroborating this. For $z=1$, we see that $\frac{\eta}{s}=\mathcal{D} T=\frac{1}{4 \pi}$ as in (2.22). It is worth noting that these quasinormal modes are diffusive damped modes. Our analysis and results hold in the regime (2.11) so in particular $\frac{q}{T^{1 / z}} \ll 1$. The quasinormal mode frequency (2.31) can then be expressed as $\omega \sim-i\left(\frac{q}{T^{1 / z}}\right)^{2} T$, and we are working at finite temperature in the hydrodynamic low frequency, low momentum regime. Then the time dependence of these modes is $\sim e^{-i \omega t} \sim e^{-\Gamma t}$, damped on long timescales.

$\boldsymbol{z}=\boldsymbol{d}_{\boldsymbol{i}}+\mathbf{2} \boldsymbol{-} \boldsymbol{\theta}$. Here, the integral in (2.29) gives the solution to (2.9) to first order as

$$
\mathcal{H}(r)=C_{0} f(r)^{-\frac{i \Omega}{2}}\left[1+\frac{i q^{2}}{\omega} r_{0}^{z-2} \log \frac{1}{r_{0} r}\right]
$$

where (2.2) now gives $T=\frac{z-1}{2 \pi} r_{0}^{z}$. Then defining $\Lambda=\frac{z-1}{2 \pi} \frac{1}{r_{c}^{z}}$ gives the low-lying quasinormal frequency

$$
\omega=-i q^{2} \cdot \frac{1}{z}\left(\frac{2 \pi}{z-1}\right)^{1-2 / z} \cdot T^{\frac{z-2}{z}} \log \frac{\Lambda}{T} \equiv-i \mathcal{D} q^{2} .
$$

This gives the shear diffusion constant $\mathcal{D}=\frac{1}{z}\left(\frac{2 \pi}{z-1}\right)^{1-2 / z} \cdot T^{\frac{z-2}{z}} \log \frac{\Lambda}{T}$ scaling logarithmically with temperature alongwith a power-law pre-factor. This also agrees with the results in $[25$, 27]. The logarithmic scaling necessitating the ultraviolet scale $\Lambda$ perhaps suggests that this leading relation for the quasinormal mode frequency is subject to subleading corrections and possibly appropriate resummations. Nevertheless, recasting as $\omega \sim-i\left(\frac{q}{T^{1 / z}}\right)^{2} T \log \frac{\Lambda}{T}$ shows that in the hydrodynamic regime $\frac{q}{T^{1 / z}} \ll 1$, this leading mode is diffusive with damped time-dependence: in fact for $T \ll \Lambda$, the extra $\log$-factor leads to additional damping. The hvLif theories arising from null reductions of AdS and nonconformal brane plane waves $[9,10,13]$ have exponents satisfying $z=d_{i}+2-\theta$ : taking the quasinormal modes as a measure of stability of the backgrounds, we see that the diffusive frequencies suggest that low lying modes do not indicate any instability. The logarithmic behaviour of the leading shear diffusion constant then suggests a possibly novel limit of hydrodynamics in these theories, perhaps stemming from the large boost in the above string constructions.

$\boldsymbol{z}>\boldsymbol{d}_{\boldsymbol{i}}+\mathbf{2}-\boldsymbol{\theta}$. The integral in $(2.29)$ scales as $r_{c}^{d_{i}+2-\theta-z}$ thus acquiring dominant (divergent) contribution from high energy scales near $r_{c} \sim 0$. There is no universal low energy behaviour emerging from near horizon physics: it appears that these methods fail 
to yield insight on quasinormal modes, as does the membrane paradigm approach [25, 27]. This sector includes e.g. reductions of $D 6$-branes $\left(d_{i}=6, z=1, \theta=9\right)$ with ill-defined asymptotics.

\subsubsection{Solving for gauge field perturbation $a_{y}$}

Using the ansatze (2.23) and rescaling $\boldsymbol{\Omega} \rightarrow \lambda \boldsymbol{\Omega}$ and $\mathbf{Q} \rightarrow \lambda \mathbf{Q}$ we can recast (2.10) as

$$
\begin{aligned}
G^{\prime \prime} & -\frac{i \lambda \boldsymbol{\Omega}}{2} \cdot \frac{f^{\prime}}{f} G^{\prime}+\partial_{r} \ln f r^{d_{i}+3-z-\theta} G^{\prime}-\frac{i \lambda \boldsymbol{\Omega}}{2}\left(\frac{f^{\prime}}{f}\right)^{\prime} G-\frac{\lambda^{2} \boldsymbol{\Omega}^{2}}{4}\left(\frac{f^{\prime}}{f}\right)^{2} G \\
& -\frac{i \lambda \boldsymbol{\Omega}}{2} \frac{f^{\prime}}{f} \partial_{r} \ln f r^{d_{i}+3-z-\theta} G+\lambda^{2}(2 \pi T)^{2} \frac{r^{2 z-2}}{f^{2}}\left(\boldsymbol{\Omega}^{2}-(2 \pi T)^{2 / z-2} \mathbf{Q}^{2} f r^{2-2 z}\right) G \\
& -\frac{k^{2}}{r^{2} f} G+\frac{(2 \pi T)^{1 / z-2} k \mathbf{Q} \cdot r^{\theta-z-d_{i}-1}}{\boldsymbol{\Omega}^{2}-(2 \pi T)^{2 / z-2} \mathbf{Q}^{2} f r^{2-2 z}} \cdot \frac{1}{\lambda}\left(F^{\prime}-\frac{i \lambda \boldsymbol{\Omega}}{2} \frac{f^{\prime}}{f} F\right)=0 .
\end{aligned}
$$

Plugging in the series ansatz (2.25) we can construct the perturbative solution for $a_{y}$ order-by-order. The leading order equation appears at $O\left(\frac{1}{\lambda}\right)$ and is given by $F_{0}^{\prime}=0$ giving $F_{0}=$ const: this can be seen to be consistent with (2.26). We will subsequently see that $G_{0}$ is determined by $F_{1}$ and $F_{0}$ while $G_{1}$ is determined by $F_{2}$ and $G_{0}$, and so on. More generally, all subsequent equations involve more variables so there is no inconsistency in the solutions due to potential overconstraining in this system of equations.

Gathering all terms of $O\left(\lambda^{0}\right)$, we see $G_{0}$ follows the equation:

$$
G_{0}^{\prime \prime}+\partial_{r} \log f r^{d_{i}+3-z-\theta} G_{0}^{\prime}-\frac{k^{2}}{r^{2} f} G_{0}+\frac{(2 \pi T)^{1 / z-2} k \mathbf{Q} \cdot r^{\theta-z-d_{i}-1}}{\boldsymbol{\Omega}^{2}-(2 \pi T)^{2 / z-2} \mathbf{Q}^{2} f r^{2-2 z}}\left(F_{1}^{\prime}-\frac{i \boldsymbol{\Omega}}{2} \frac{f^{\prime}}{f} F_{0}\right)=0
$$

For $z<d_{i}+2-\theta$, using (2.26) and (2.29) we can write the most general solution to the above equation in terms of a new radial variable as

$$
G_{0}(x)=\frac{A}{m n}+C_{1} x_{2}^{n} F_{1}\left[2, \frac{n}{m}, 2+\frac{n}{m} ; x^{m}\right]-\frac{C_{2}}{n} x^{-m}\left(n+(m-n) x^{m}\right),
$$

where

$$
x=r_{0} r, \quad m=d_{i}+z-\theta, \quad n=2 z-2, \quad A=i C_{0} k r_{0}^{d_{i}-\theta} \frac{q}{\omega},
$$

while $C_{1}$ and $C_{2}$ are arbitrary constants which are to be fixed by demanding regularity of $a_{y}$ at the horizon. Potential divergences in $a_{y}(r)$ and $a_{y}^{\prime}(r)$ near the horizon can be removed by choosing $C_{1}=0$ and $C_{2}=\frac{A}{m^{2}}$. In terms of the original radial coordinate $r$, the solution is

$$
G_{0}(r)=-i C_{0} k \frac{q}{\omega} \cdot \frac{r_{0}^{d_{i}-\theta}}{\left(d_{i}+z-\theta\right)^{2}} \cdot\left(r_{0} r\right)^{-\left(d_{i}+z-\theta\right)} f(r) .
$$

For relativistic theories, $(z=1, k=0)$ the above expression vanishes identically implying that the shear mode sector is governed exclusively by metric perturbations $H_{x y}$ and $H_{t y}$.

The subleading term in $a_{y}$ i.e. $G_{1}(r)$ can be determined by collecting terms of $O(\lambda)$ from (2.36). The inhomogeneous part of the equation governing $G_{1}$ involves $F_{2}^{\prime}$ and $F_{1}$. $F_{2}^{\prime}(r)$ can be evaluated from $O\left(\lambda^{2}\right)$ terms of (2.24). Although we could find the general solution to $G_{1}$, finding the integration constants respecting regularity at the horizon seems difficult and cumbersome by analytic means. We discuss further details about $F_{2}$ and $G_{1}$ in appendix B. 


\section{Dual field theory correlation functions}

In this section, we will determine the energy-momentum tensor correlation functions $\langle T T\rangle$ following the prescription in [33, 34], and defining $T_{\mu \nu}$ as dual to the perturbation $h_{\mu \nu}$. The action governing the perturbations using the variables $H_{t y}, H_{x y}$ and $a_{y}$ in (2.3) is given by

$$
\begin{aligned}
S^{\text {pert }}= & -\frac{1}{16 \pi G_{N}^{\left(d_{i}+2\right)}} \int d^{d_{i}+2} x\left[\frac { 1 } { 2 } r ^ { \theta - z - d _ { i } + 1 } \left(-r^{2 z-2}\left(H_{t y}^{\prime}\right)^{2}+\frac{r^{2 z-2}}{f}\left(\omega H_{x y}+q H_{t y}\right)^{2}\right.\right. \\
& \left.\left.+f\left(H_{x y}^{\prime}\right)^{2}\right)-k H_{t y} a_{y}^{\prime}+\frac{1}{2} r^{d_{i}+3-z-\theta} f\left(a_{y}^{\prime}\right)^{2}+\frac{1}{2} r^{d_{i}+3-z-\theta}\left(\frac{r^{2 z-2}}{f} \omega^{2}-q^{2}\right) a_{y}^{2}\right] .
\end{aligned}
$$

The equations of motion from this action lead to (2.4)-(2.7) and we have suppressed contact terms. The above expression can be recast as a bulk piece (which vanishes by the equations of motion) and a boundary term. This boundary action takes the form

$$
S^{\text {bdy }}=-\frac{1}{32 \pi G_{N}^{\left(d_{i}+2\right)}} \int d^{d_{i}+1} x\left[r^{\theta-z-d_{i}+1}\left(-r^{2 z-2} H_{t y} H_{t y}^{\prime}+f H_{x y} H_{x y}^{\prime}\right)+r^{d_{i}+3-z-\theta} a_{y} a_{y}^{\prime}\right]+\cdots
$$

again suppressing contact terms. Using the equation of motion (2.6) and the definition of $\mathcal{H}(2.8)$, we can recast the relevant terms of the above action as

$$
S^{\text {bdy }}=\lim _{r \rightarrow r_{c}}-\frac{1}{32 \pi G_{N}^{\left(d_{i}+2\right)}} \int d^{d_{i}+1} x\left[\frac{f r^{\theta-z-d_{i}+1}}{\omega^{2}-q^{2} f r^{2-2 z}} \mathcal{H}^{\prime}(r, x) \mathcal{H}(r, x)\right]+\cdots
$$

In the above equation, we have exhibited only those terms that contribute to the 2-point function i.e. terms that are at least second order in $\mathcal{H}$. Using the Fourier decomposition of the bulk field as $\mathcal{H}(r, t, x)=\int d \omega d q e^{-i \omega t+i q x} \mathcal{H}(r, \omega, q)$ we can further recast as

$$
S^{\text {bdy }}=\lim _{r \rightarrow r_{c}}-\frac{1}{32 \pi G_{N}^{\left(d_{i}+2\right)}} \int d \omega d q \frac{f r^{\theta-z-d_{i}+1}}{\omega^{2}-q^{2} f r^{2-2 z}} \mathcal{H}^{\prime}(r, \omega, q) \mathcal{H}(r,-\omega,-q) .
$$

For $z=1, \theta=0$, this agrees with the AdS case in [28]. Also, for theories with Lifshitz symmetry, it is clear that (3.4) is Lifshitz-invariant. The $\langle T T\rangle$ shear correlator in the boundary theory is defined as

$$
G_{x y, x y}=\left\langle T_{x y} T_{x y}\right\rangle=\left.\frac{\delta^{2} S^{\mathrm{bdy}}}{\delta h_{y}^{x(0)} \delta h_{y}^{x(0)}}\right|_{r \approx r_{c}} .
$$

We define the boundary fields through the $r \rightarrow r_{c} \sim 0$ limits of the bulk fields, i.e. $\mathcal{H}^{(0)}(\omega, q)=\mathcal{H}\left(r_{c}, \omega, q\right)$ and $h_{y}^{x(0)}(\omega, q)=h_{y}^{x(0)}\left(r_{c}, \omega, q\right)$. The asymptotics can be analysed by studying (2.9) in the limit $r \rightarrow 0$ at zero momenta and frequency $(\mathbf{Q}=\boldsymbol{\Omega}=0)$ i.e.

$$
\mathcal{H}^{\prime \prime}+\frac{z+\theta-d_{i}-1}{r} \mathcal{H}^{\prime}=0 .
$$

The solutions are $\mathcal{H}=A r^{\Delta}$ with $\Delta=0, d_{i}+2-z-\theta$. Thus in the hydrodynamic regime i.e. $\boldsymbol{\Omega}, \mathbf{Q} \ll 1$, we can schematically write the mode as $\mathcal{H}=\mathcal{A}(\omega, q)+\mathcal{B}(\omega, q) r^{d_{i}+2-z-\theta}$ where the functions $\mathcal{A}(\omega, q)$ and $\mathcal{B}(\omega, q)$ can be read off from (2.30),

$$
\mathcal{A}(\omega, q)=C_{0}\left[1+i \frac{q^{2}}{\omega} \frac{r_{0}^{z-2}}{d_{i}+2-z-\theta}\right], \quad \mathcal{B}(\omega, q)=-C_{0} \frac{i q^{2}}{\omega} \frac{r_{0}^{d_{i}-\theta}}{d_{i}+2-z-\theta} .
$$


We can write the normalized bulk field $\mathcal{H}$ in terms of its source $\mathcal{H}^{0}$

$$
\mathcal{H}(\omega, q)=\mathcal{H}^{(0)}(\omega, q) \frac{1}{\mathcal{N}}\left[1+\frac{\mathcal{B}(\omega, q)}{\mathcal{A}(\omega, q)} r^{d_{i}+2-z-\theta}\right], \quad \mathcal{N}=1+\frac{\mathcal{B}(\omega, q)}{\mathcal{A}(\omega, q)} r_{c}^{d_{i}+2-z-\theta}
$$

Note that the normalization factor $\mathcal{N}$ satisfies $\mathcal{N} \sim 1$ as $r_{c} \rightarrow 0$ for $z<2+d_{i}-\theta$. Using this solution in (3.4), we get

$$
S^{\mathrm{bdy}}=\lim _{r \rightarrow r_{c}}-\int \frac{d \omega d q}{32 \pi G_{N}^{\left(d_{i}+2\right)}} \frac{f r^{2-2 z}}{\omega^{2}-q^{2} f r^{2-2 z}} \cdot \frac{d_{i}+2-z-\theta}{\mathcal{N}} \cdot \frac{\mathcal{B}(\omega, q)}{\mathcal{A}(\omega, q)} \cdot \mathcal{H}^{(0)}(\omega, q) \mathcal{H}^{(0)}(-\omega,-q)
$$

As $r \rightarrow r_{c} \rightarrow 0$ with $z>1$, we note that $\lim _{r \rightarrow 0} \frac{f r^{2-2 z}}{\omega^{2}-q^{2} f r^{2-2 z}} \frac{1}{\mathcal{N}^{2}} \frac{\mathcal{B}(\omega, q)}{\mathcal{A}(\omega, q)}=-\frac{1}{q^{2}} \frac{\mathcal{B}(\omega, q)}{\mathcal{A}(\omega, q)}$. From the definition of $\mathcal{H}$ in (2.8) and also noting $H_{x y} \equiv h_{y}^{x}=h_{x}^{y}$ and $H_{t y} \equiv h_{t}^{y}$ from (2.3), we see that $\frac{\delta}{\delta H_{x y}^{(0)}}=\frac{\delta}{\delta h_{y}^{x(0)}}=\omega \frac{\delta}{\delta \mathcal{H}^{(0)}}$. Thus the correlation function (3.5) becomes

$$
\begin{aligned}
G_{x y, x y}=\left\langle T_{y}^{x}(k) T_{y}^{x}(-k)\right\rangle=\frac{\delta^{2} S^{\mathrm{bdy}}}{\delta h_{y}^{x(0)}(k) \delta h_{y}^{x(0)}(-k)} & =\omega^{2} \frac{\delta^{2} S^{\text {bdy }}}{\delta \mathcal{H}^{(0)}(k) \delta \mathcal{H}^{(0)}(-k)} \\
& =\frac{1}{16 \pi G_{N}^{\left(d_{i}+2\right)}} \frac{i \omega^{2} r_{0}^{d_{i}-\theta}}{\omega+i \mathcal{D} q^{2}},
\end{aligned}
$$

with $\mathcal{D}$ given in (2.32), and $k=(\omega, q)$. The Kubo formula then gives viscosity as

$$
\eta=\lim _{\omega \rightarrow 0} \frac{G_{x y, x y}(\omega, q=0)}{i \omega}=\frac{r_{0}^{d_{i}-\theta}}{16 \pi G_{N}^{\left(d_{i}+2\right)}} .
$$

With the entropy density given in terms of the horizon area $s=\frac{r_{0}^{d_{i}-\theta}}{4 G_{N}^{\left(d_{i}+2\right)}}$, we obtain universal behaviour for the viscosity bound $\frac{\eta}{s}=\frac{1}{4 \pi}$, as for relativistic theories [14]. This is consistent with $[25,27]$, where we conjectured the universal relation (2.33) saturating the proposed viscosity bound in [14]. Also, we can write down other correlators as follows:

$$
\begin{aligned}
G_{t y, t y} & =\left\langle T_{t}^{y}(k) T_{t}^{y}(-k)\right\rangle=\frac{1}{16 \pi G_{N}^{\left(d_{i}+2\right)}} \frac{i q^{2} r_{0}^{d_{i}-\theta}}{\omega+i \mathcal{D} q^{2}}, \\
G_{t y, x y} & =\left\langle T_{t}^{y}(k) T_{x}^{y}(-k)\right\rangle=\frac{1}{16 \pi G_{N}^{\left(d_{i}+2\right)}} \frac{i \omega q r_{0}^{d_{i}-\theta}}{\omega+i \mathcal{D} q^{2}} .
\end{aligned}
$$

Each correlator above exhibits a pole at $\omega=-i \mathcal{D} q^{2}$ which is the lowest lying quasinormal mode as we have seen earlier. The viscosity (3.11) above agrees with the result in [23]: however what is noteworthy in our analysis is that this is obtained in the regime $z<$ $d_{i}+2-\theta$.

\subsection{Dual field theory correlation functions: $z=d_{i}+2-\theta$}

hvLif theories with $z=d_{i}+2-\theta$ arise from the null reductions of highly boosted black branes in $[9,13]$ as mentioned previously. The asymptotic fall-offs in (3.6), (3.7), coincide in this case: this is the interface of the standard/alternative quantization in [35], and one of the solutions contains a logarithm. We see that in this case, (3.6) reduces to

$$
\mathcal{H}^{\prime \prime}+\frac{1}{r} \mathcal{H}^{\prime}=0
$$


with the solution $\mathcal{H}=\mathcal{A}(\omega, q)+\mathcal{B}(\omega, q) \log \frac{1}{r_{0} r}$ in (2.34). We define the normalized bulk field in terms of the source as

$$
\mathcal{H}(r, \omega, q)=\mathcal{H}_{0}(\omega, q) \cdot \frac{1+\frac{i q^{2}}{\omega} r_{0}^{z-2} \log \frac{1}{r_{0} r}}{1+\frac{i q^{2}}{\omega} r_{0}^{z-2} \log \frac{1}{r_{0} r_{c}}},
$$

the source being the boundary value $\mathcal{H}^{(0)}(\omega, q)=\mathcal{H}\left(r_{c}, \omega, q\right)$. Note that unlike $\mathcal{N}$ in (3.8), the logarithm does not die near the boundary and the normalization above is less trivial. Using (3.14), the relevant part of the boundary action becomes

$$
\begin{aligned}
S^{\mathrm{bdy}} & =\lim _{r \rightarrow r_{c}}-\frac{1}{32 \pi G_{N}^{\left(d_{i}+2\right)}} \int d^{d_{i}+1} x\left[\frac{f r^{3-2 z}}{\omega^{2}-q^{2} f r^{2-2 z}} \mathcal{H}^{\prime}(r, x) \mathcal{H}(r, x)\right] \\
& =\frac{1}{32 \pi G_{N}^{\left(d_{i}+2\right)}} \int d \omega \cdot d q \frac{i r_{0}^{z-2}}{\omega} \frac{1}{\left(1+\frac{i q^{2}}{\omega} r_{0}^{z-2} \log \frac{1}{r_{0} r_{c}}\right)} \mathcal{H}^{(0)}(\omega, q) \mathcal{H}^{(0)}(-\omega,-q) .
\end{aligned}
$$

As before, we obtain the energy-momentum tensor correlation functions as

$$
\begin{aligned}
G_{x y, x y}=\left\langle T_{y}^{x}(k) T_{y}^{x}(-k)\right\rangle=\frac{\delta^{2} S^{\text {bdy }}}{\delta h_{y}^{x(0)}(k) \delta h_{y}^{x(0)}(-k)} & =\omega^{2} \frac{\delta^{2} S^{\text {bdy }}}{\delta \mathcal{H}^{(0)}(k) \delta \mathcal{H}^{(0)}(-k)} \\
& =\frac{1}{16 \pi G_{N}^{d_{i}+2}} \frac{i \omega^{2} r_{0}^{z-2}}{\omega+i \mathcal{D} q^{2}},
\end{aligned}
$$

using the expression for the shear diffusion constant in (2.35). Using the Kubo formula, we again see that

$$
\eta=\lim _{\omega \rightarrow 0} \frac{G_{x y, x y}(\omega, q=0)}{i \omega}=\frac{r_{0}^{z-2}}{16 \pi G_{N}^{\left(d_{i}+2\right)}}=\frac{r_{0}^{d_{i}-\theta}}{16 \pi G_{N}^{\left(d_{i}+2\right)}} .
$$

Note that the diffusive pole again coincides with the quasinormal mode frequency in (2.35): note that this here arises from the nontrivial normalization factor in (3.14). Now using the entropy density $s=\frac{r_{0}^{d_{i}-\theta}}{4 G_{N}^{\left(d_{i}+2\right)}}$ given in terms of the horizon area, we again find universal behaviour $\frac{\eta}{s}=\frac{1}{4 \pi}$ for the viscosity bound, again as for relativistic theories [14]. It is worth noting that in applying the Kubo formula, we first restrict to the zero momentum sector $q=0$, which kills off the term containing the leading diffusion constant $\mathcal{D}$ which, strictly speaking, is logarithmically divergent as $r_{c} \rightarrow 0$.

\section{Discussion}

We have studied low lying hydrodynamic quasinormal modes for shear perturbations of hyperscaling violating Lifshitz black branes: these are of the form $\omega=-i \mathcal{D} q^{2}$ where $\mathcal{D}$ is the shear diffusion constant. This is consistent with $\mathcal{D}$ obtained in $[25,27]$ through a membrane-paradigm analysis of near horizon perturbations and the associated shear diffusion equation. This shear diffusion equation $\partial_{t} j^{t}=\mathcal{D} \partial_{x}^{2} j^{t}$ following from the second order Einstein equations in a sense dictates the form of $\mathcal{D}$ above and is consistent with the low-lying quaisnormal mode spectrum. The analysis here and the associated boundary conditions are valid for theories with exponents satisfying $z \leq d_{i}+2-\theta$ : this is the regime that is continuously connected to AdS theories $(z=1, \theta=0)$. Using the asymptotics 
of these quasinormal modes, retarded correlators of dual operators can be obtained: we have seen that the poles of the retarded $\langle T T\rangle$ correlator at finite temperature coincide with the lowest quasinormal frequencies of the dual gravity theory. This analysis appears consistent with the Kubo formula for viscosity via the retarded Green's function at zero momentum only for theories with $z \leq d_{i}+2-\theta$. Perhaps this is not surprising given the asymptotic fall-offs of the quasinormal modes: for $z>d_{i}+2-\theta$, high energy modes appear to dominate, with no universal low energy behaviour for the diffusion expression. It would be interesting to understand this better.

hvLif spacetimes with $z=d_{i}+2-\theta$ exhibit more interesting hydrodynamic behaviour as we have seen: the asymptotic fall-offs of the bulk modes coincide here. While correlation functions in the Kubo limit continue to reveal universal behaviour $\frac{\eta}{s}=\frac{1}{4 \pi}$ for the viscosity, the leading shear diffusion constant exhibits logarithmic scaling (involving the ultraviolet cutoff), perhaps suggesting that subleading contributions are important with some resummation required. The null reductions $[9,13]$ of highly boosted black branes give hvLif theories with $z=d_{i}+2-\theta$. It is worth noting that the boost induces anisotropy in the system (although the lower dimensional theory after compactification enjoys translation invariance in the spatial directions so that the zero momentum Kubo limit studied here is unambiguous). The large boost involved in these string constructions possibly leads to novel hydrodynamic behaviour. It would perhaps be interesting to study this directly from the null reduction of black brane hydrodynamics and the fluid/gravity correspondence [36].

\section{Acknowledgments}

It is a pleasure to thank Kedar Kolekar, Alok Laddha, R. Loganayagam and Mukund Rangamani for helpful discussions. DM thanks the organizers of the ICTP Spring School on Superstring Theory, Trieste, Italy, and the Asian Winter School on Strings, Particles and Cosmology, Sun Yat-sen University, China, and the String Theory Groups at TIFR Mumbai, HRI Allahabad, and ICTS Bangalore, for hospitality while this work was in progress. This work is partially supported by a grant to CMI from the Infosys Foundation.

\section{A Reviewing hyperscaling violating Lifshitz spacetimes}

The metric (2.1) is a solution to the Einstein-Maxwell-Dilaton action

$$
S=-\frac{1}{16 \pi G_{N}^{(d+1)}} \int d^{d+1} x \sqrt{-G}\left[R-\frac{1}{2} \partial_{\mu} \phi \partial^{\mu} \phi-\frac{Z(\phi)}{4} F_{\mu \nu} F^{\mu \nu}+V(\phi)\right],
$$

where the various fields and parameters appearing in the action are listed as follows:

$$
\begin{aligned}
\phi & =\sqrt{2\left(d_{i}-\theta\right)\left(z-\theta / d_{i}-1\right)} \log r, \\
A_{t} & =\frac{\alpha f(r)}{r^{d_{i}+z-\theta}}, \quad \alpha=-\sqrt{\frac{2(z-1)}{d_{i}+z-\theta}}, \quad A_{i}=0 . \\
V(\phi) & =\left(d_{i}+z-\theta\right)\left(d_{i}+z-\theta-1\right) r^{-\frac{2 \theta}{d_{i}}} ; \quad Z(\phi)=r^{\frac{2 \theta}{d_{i}}+2 d_{i}-2 \theta}=e^{\lambda \phi} .
\end{aligned}
$$


The null energy conditions following from (2.1) give constraints on the Lifshitz $z$ and hyperscaling violating $\theta$ exponents

$$
(z-1)\left(d_{i}+z-\theta\right) \geq 0, \quad\left(d_{i}-\theta\right)\left(d_{i}(z-1)-\theta\right) \geq 0 .
$$

Varying with $G_{\mu \nu}, A_{\mu}$ and $\phi$, we obtain the following equations of motion,

$$
\begin{gathered}
R_{\mu \nu}=\frac{1}{2} \partial_{\mu} \phi \partial_{\nu} \phi-G_{\mu \nu} \frac{V(\phi)}{d-1}+\frac{Z(\phi)}{2} G^{\rho \sigma} F_{\rho \mu} F_{\sigma \nu}-\frac{Z(\phi)}{4(d-1)} G_{\mu \nu} F_{\rho \sigma} F^{\rho \sigma}, \\
\nabla_{\mu}\left(Z(\phi) F^{\mu \nu}\right)=0, \quad \frac{1}{\sqrt{-G}} \partial_{\mu}\left(\sqrt{-G} G^{\mu \nu} \partial_{\nu} \phi\right)+\frac{\partial V(\phi)}{\partial \phi}-\frac{1}{4} \frac{\partial Z(\phi)}{\partial \phi} F_{\rho \sigma} F^{\rho \sigma}=0 .
\end{gathered}
$$

Turning on gravitational, gauge field and scalar field perturbations $h_{\mu \nu}(x), a_{\mu}(x)$ and $\varphi(x)$, the linearized Einstein's equations are given by

$$
\begin{aligned}
R_{\mu \nu}^{(1)}= & \frac{1}{2} \partial_{\mu} \phi \partial_{\nu} \varphi+\frac{1}{2} \partial_{\mu} \varphi \partial_{\nu} \phi-\frac{V}{2}\left(h_{\mu \nu}-G_{\mu \nu} \delta \varphi\right) \\
& +\frac{Z}{2}\left[G^{\rho \sigma} F_{\mu \rho} f_{\nu \sigma}+G^{\rho \sigma} f_{\mu \rho} F_{\nu \sigma}-h^{\rho \sigma} F_{\mu \rho} F_{\nu \sigma}+\lambda \varphi G^{\rho \sigma} F_{\mu \rho} F_{\nu \sigma}\right] \\
& -Z\left[\frac{1}{4} G_{\mu \nu}\left(F_{\rho \sigma} f^{\rho \sigma}-g^{\rho \alpha} h^{\sigma \beta} F_{\rho \sigma} F_{\alpha \beta}\right)+\frac{1}{8} h_{\mu \nu} F_{\rho \sigma} F^{\rho \sigma}+\frac{1}{8} \lambda \varphi G_{\mu \nu} F_{\rho \sigma} F^{\rho \sigma}\right],
\end{aligned}
$$

where

$$
\begin{aligned}
R_{\mu \nu}^{(1)} & =\frac{1}{2}\left[\nabla_{\alpha} \nabla_{\nu} h_{\mu}^{\alpha}+\nabla_{\alpha} \nabla_{\mu} h_{\nu}^{\alpha}-\nabla_{\alpha} \nabla^{\alpha} h_{\mu \nu}-\nabla_{\nu} \nabla_{\mu} h\right] ; \\
f_{\mu \nu} & =\partial_{\mu} a_{\nu}-\partial_{\nu} a_{\mu} ; \quad h=G^{\mu \nu} h_{\mu \nu} ; \quad \delta=\frac{2 \theta / d_{i}}{\sqrt{2\left(d_{i}-\theta\right)\left(z-\theta / d_{i}-1\right)}} .
\end{aligned}
$$

Similarly, the linearized Maxwell Equations (A.7) are

$$
\nabla_{\mu}\left(Z f^{\mu \nu}\right)-\nabla_{\mu}\left(Z h^{\mu \rho} F_{\rho}{ }^{\nu}\right)-Z\left(\nabla_{\mu} h^{\nu \sigma}\right) F_{\sigma}^{\mu}+\frac{1}{2}\left(\nabla_{\mu} h\right) Z F^{\mu \nu}+\lambda Z F^{\mu \nu} \partial_{\mu} \varphi=0 .
$$

The linearized scalar field equation is:

$$
\begin{aligned}
\frac{1}{\sqrt{-G}} \partial_{\mu}\left(\sqrt{-G} G^{\mu \nu} \partial_{\nu} \varphi\right) & -\frac{1}{\sqrt{-G}} \partial_{\mu}\left(\sqrt{-G} h^{\mu \nu} \partial_{\nu} \phi\right)+\frac{1}{2} G^{\mu \nu} \partial_{\nu} \phi \partial_{\mu} h+V \delta^{2} \varphi \\
& -\frac{\lambda Z}{4}\left(2 F_{\mu \nu} f^{\mu \nu}-2 G^{\mu \rho} h^{\nu \sigma} F_{\mu \nu} F_{\rho \sigma}+\lambda \varphi F_{\mu \nu} F^{\mu \nu}\right)=0 .
\end{aligned}
$$

In (A.8), (A.10), (A.11), indices are raised and lowered using (2.1).

\section{B Solution for gauge field perturbation $a_{y}$ : details}

$\boldsymbol{G}_{\mathbf{0}}(\boldsymbol{r})$ : using (2.17) and (2.20) and the variables in (2.39), we can simplify (2.37) to

$$
\frac{d^{2} G_{0}}{d x^{2}}+\left(\frac{2 m-n+1}{x}-\frac{m}{x\left(1-x^{m}\right)}\right) \frac{d G_{0}}{d x}-\frac{m n}{x^{2}\left(1-x^{m}\right)} G_{0}+\frac{A}{x^{2}\left(1-x^{m}\right)}=0 .
$$

$G_{0}(r)$ in $(2.38)$ can then be obtained as the solution to the above equation. 
$\boldsymbol{F}_{\mathbf{2}}(\boldsymbol{r}, \boldsymbol{t}, \boldsymbol{x})$ solution. The equation governing $F_{2}(r)$ follows from $O\left(\lambda^{2}\right)$ terms of $(2.24)$. The relevant terms following from the first two lines of (2.24) are computationally straightforward to derive. Let us concentrate on the last line of (2.24). Concentrating on the powers of $\lambda$, the integral in the last term can be integrated by parts and rewritten as

$$
\begin{aligned}
\lambda^{3} f^{\frac{i \lambda \Omega}{2}} \int d s f^{-\frac{i \lambda \Omega}{2}} s^{d_{i}+1-z-\theta} G \approx \lambda^{3} f^{\frac{i \lambda \Omega}{2}}\left[f^{-\frac{i \lambda \Omega}{2}} \int d s s^{d_{i}+1-z-\theta} G_{0}\right. \\
\left.+\frac{i \lambda \Omega}{2} \int d s f^{-\frac{i \lambda \Omega}{2}} \frac{f^{\prime}}{f} \int d s^{\prime} s^{\prime d_{i}+1-z-\theta} G_{0}\right] \sim O\left(\lambda^{3}\right)+O\left(\lambda^{4}\right) .
\end{aligned}
$$

The above expression shows that the leading contribution from the last term of (2.24) becomes relevant at $O\left(\lambda^{3}\right)$ and has no role to play in determining $F_{2}(r)$. We can write the equation governing $F_{2}$ as

$$
\begin{aligned}
F_{2}^{\prime \prime}-\frac{H^{\prime}}{H} F_{2}^{\prime}+\left[-i \boldsymbol{\Omega} \frac{f^{\prime}}{f} F_{1}^{\prime}-\right. & \frac{i \boldsymbol{\Omega}}{2}\left(\frac{f^{\prime}}{f}\right)^{\prime} F_{1}-\frac{\boldsymbol{\Omega}^{2}}{4}\left(\frac{f^{\prime}}{f}\right)^{2} C_{0}+\frac{i \boldsymbol{\Omega}}{2} \frac{f^{\prime}}{f} \frac{H^{\prime}}{H} F_{1} \\
& \left.+(2 \pi T)^{2} \frac{r^{2 z-2}}{f^{2}} \cdot\left(\boldsymbol{\Omega}^{2}-(2 \pi T)^{2 / z-2} \mathbf{Q}^{2} f r^{2-2 z}\right) C_{0}\right]=0 .
\end{aligned}
$$

Using (2.29) and integrating the above once, we get

$$
\begin{aligned}
F_{2}^{\prime}(r)= & \frac{i \boldsymbol{\Omega}}{2} \frac{f^{\prime}}{f} F_{1}-H \tilde{C}_{1} \\
& -\frac{C_{0} m^{2} r_{0}^{m}}{4}\left(\frac{\left(r_{0} r\right)^{n+2-m}}{n+2-m}{ }_{2} F_{1}\left[1, \frac{n+2-m}{m}, \frac{n+2}{m},\left(r_{0} r\right)^{m}\right]+\frac{1}{m} \log f\right) \cdot H
\end{aligned}
$$

where $\bar{C}_{1}$ is an arbitrary integration constant. Choosing $\tilde{C}_{1}=\frac{C_{0}}{4} m r_{0}^{m}\left(\gamma+\psi\left(\frac{n+2}{m}\right)\right)$ where $\gamma$ is the Euler-Mascheroni constant and $\psi$ is the digamma function, ensures $F_{2}^{\prime}(r)$ is finite at the horizon (for $z<d_{i}+2-\theta$ ). The above expression can be integrated again subject to the boundary condition $F_{2}\left(r \sim \frac{1}{r_{0}}\right)=0$ to obtain an explicit expression for the function $F_{2}(r)$.

$\boldsymbol{G}_{\mathbf{1}}(\boldsymbol{r}, \boldsymbol{t}, \boldsymbol{x})$ solution. Collecting $O(\lambda)$ terms from (2.36) gives the equation governing $G_{1}(r)$,

$$
\begin{aligned}
& G_{1}^{\prime \prime}+\partial_{r} \log f r^{d_{i}+3-z-\theta} G_{1}^{\prime}-\frac{k^{2}}{r^{2} f} G_{1}=\frac{i \boldsymbol{\Omega}}{2} \frac{f^{\prime}}{f}\left(G_{0}^{\prime}+\partial_{r} \log f r^{d_{i}+3-z-\theta} G_{0}\right)+\frac{i \boldsymbol{\Omega}}{2}\left(\frac{f^{\prime}}{f}\right)^{\prime} G_{0} \\
& +(2 \pi T)^{\frac{1}{z}-2} \frac{\mathbf{Q} k}{r^{2} f}\left(\frac{C_{0}}{4} m^{2} r_{0}^{m}\left[\frac{\left(r_{0} r\right)^{n+2-m}}{n+2-m}{ }_{2} F_{1}\left[1, \frac{n+2-m}{m}, \frac{n+2}{m} ;\left(r_{0} r\right)^{m}\right]+\frac{1}{m} \log f\right]+\tilde{C}_{1}\right)
\end{aligned}
$$

The homogeneous part of the above equation is identical to the homogeneous part of (2.37). This helps us in writing down the solution for $G_{1}$ as

$$
G_{1}(x)=\bar{C}_{1} y_{1}(x)-y_{1}(x) \int \frac{h(x) y_{2}(x)}{W(x)} d x+y_{2}(x) \int \frac{h(x) y_{1}(x)}{W(x)} d x
$$


where $x$ is the radial variable defined in (2.39). Also,

$$
\begin{aligned}
y_{1}(x) & =x^{-m} \frac{n+(m-n) x^{m}}{n}, \\
y_{2}(x) & =x^{n}{ }_{2} F_{1}\left[2, \frac{n}{m}, 2+\frac{n}{m} ; x^{m}\right], \\
W(x) & =\frac{(m+n) x^{n-m-1}}{1-x^{m}}, \\
h(x) & =\frac{\Lambda_{1}}{x^{2}}+\frac{\Lambda_{2}}{x^{2}\left(1-x^{m}\right)}+\frac{\Lambda}{x^{2}\left(1-x^{m}\right)}\left[\frac{x^{n+2-m}}{n+2-m}{ }_{2} F_{1}\left[1, \frac{n+2-m}{m}, \frac{n+2}{m} ; x^{m}\right]+\frac{\log f}{m}\right]
\end{aligned}
$$

where the constants $\Lambda_{1}, \Lambda_{2}$ and $\Lambda$ are given by $\Lambda_{1}=-\frac{\Lambda}{m}\left(2-\frac{n}{m}\right), \Lambda_{2}=\Lambda\left(\gamma+\psi\left(\frac{2+n-m}{m}\right)\right)$, $\Lambda=C_{0} k q r_{0}^{m-n-2}$. In principle we can fix the constant $\bar{C}_{1}$ by demanding regularity of the solution near horizon i.e. as $x \sim 1$. In practice, this appears difficult analytically.

The crucial thing to note about the $a_{y}$ solution is that even the leading order piece $G_{0} \sim \frac{q}{\omega}$ vanishes in the zero momentum sector i.e. when $q=0$. However, Kubo's formula for response functions (the $\left\langle a_{y}(k) a_{y}(-k)\right\rangle$ correlator here) is strictly evaluated at zero momentum: this leads to $\left\langle a_{y}(k) a_{y}(-k)\right\rangle=0$. This is similar to the behaviour of the correlators $\left\langle T_{t}^{y}(k) T_{t}^{y}(-k)\right\rangle$ and $\left\langle T_{t}^{y}(k) T_{x}^{y}(-k)\right\rangle$ given by (3.12) which vanishes in the $q \rightarrow 0$ limit. Indeed from (2.4)-(2.7) we observe that when $q=0$, the $H_{t y}$ and $a_{y}$ fields decouple and the shear mode sector is governed exclusively by the dynamics of $H_{x y}$. This results in a non-trivial $\left\langle T_{x}^{y}(k) T_{x}^{y}(-k)\right\rangle$ correlator i.e. (3.10) which is indeed non-zero in the $q=0$ sector eventually leading to (3.11).

\section{Shear diffusion and $\frac{\eta}{s}$ from membrane paradigm}

Here we briefly review [25, 27] which motivated the present work. The analysis there adapted the study in [32] of obtaining a diffusion equation from near horizon shear gravitational perturbations in a membrane paradigm type approach to hvLiv theories (2.1).

The simplest context is dilaton gravity with $A_{\mu}=0$ in (A.1) which fixes $z=1$ from (A.3). The spatial directions $\left\{x_{i}\right\}$ enjoy translation invariance. Thus the diffusion of shear gravitational modes $h_{x y}, h_{t y}$, can be mapped to charge diffusion in an auxilliary theory obtained by compactifying the $\left(d_{i}+2\right)$-dimn theory along one of the spatial directions, say $y$. We turn on plane wave modes for the perturbations $\propto e^{-\Gamma t+i q x}$ where $\Gamma$ is the typical time scale over which the perturbation decays while $q$ is the momentum along $x$. The $y$-compactification maps $h_{x y}, h_{t y}$ to gauge fields $\mathcal{A}_{t}=g_{y y}^{-1} h_{t y}$ and $\mathcal{A}_{x}=g_{y y}^{-1} h_{x y}$ (identical to the field variables in (2.3)) in the $d_{i}+1$-dimensional theory. These satisfy the Maxwell Equations i.e. $\partial_{\mu}\left(\frac{1}{g_{\text {eff }}^{2}} \sqrt{-g} \mathcal{F}^{\mu \nu}\right)=0$. The field strength is defined as usual as $\mathcal{F}_{\mu \nu}=\partial_{\mu} \mathcal{A}_{\nu}-\partial_{\nu} \mathcal{A}_{\mu}$ while the $r$-dependent coupling is $1 / g_{\text {eff }}^{2}=\left(g_{y y}\right)^{\frac{d_{i}}{d_{i}-1}}$. Then defining currents $j^{\mu}=n_{\nu} \mathcal{F}^{\mu \nu} \equiv\left(j^{t}, j^{x}\right)$ on the stretched horizon, with $n$ the outward unit normal, a set of approximations allows using these equations to eventually obtain Fick's Law $j^{x}=-\mathcal{D} \partial_{x} j^{t}$ for charge diffusion of the gauge field $\mathcal{A}_{\mu}$ in the compactified background: we then identify the diffusion constant.

In hvLif theories with a gauge field, the metric perturbations couple to the gauge field perturbations which complicates formulating Fick's Law. However, we note that these are 
uncharged black branes with the gauge field and scalar simply serving as sources supporting the nonrelativistic background. Using intuition from the fluid/gravity correspondence [36], we expect that the near horizon perturbations must be characterized simply by local temperature and velocity fluctuations. Thus since charge cannot be a parameter, we expect that the structure of the diffusion equation and the shear diffusion constant should not be dramatically altered by the presence of the gauge field. In this light, we see that (2.6) with explicit $t$ and $x$ derivatives is schematically of the form $\partial_{x}\left(\# \partial_{r} h_{x y}\right) \sim \partial_{t}\left(\# \partial_{r} h_{t y}-\# a_{y}\right)$, using (2.6). This suggests the use of new field variables $\tilde{h}_{x y}$ and $\tilde{h}_{x y}$,

$$
\tilde{h}_{t y} \equiv h_{t y}-k r^{2 \theta / d_{i}-2} \int_{r_{c}}^{r} s^{d_{i}+1-z-\theta} a_{y} d s, \quad \tilde{h}_{x y} \equiv h_{x y}
$$

or equivalently $\tilde{\mathcal{A}}_{t}$ and $\tilde{\mathcal{A}}_{x}$ in the compactified theory. This is consistent with and motivated (2.8). The field $\chi$ is an effective scalar field arising from the compactification of $a_{y}$. Defining the currents $\tilde{j}^{x}=j^{x}=n_{r} \mathcal{F}^{x r}$ and $\tilde{j}^{t}=n_{r} \tilde{\mathcal{F}}^{t r}=-n_{r} \partial^{r} \tilde{\mathcal{A}}_{t}$ on the stretched horizon we can express Fick's Law as $\tilde{j}^{x}=-\mathcal{D} \partial_{x} \tilde{j}^{t}$, which we outline below.

In the near horizon region the equations of motion simplify significantly if we make the following assumptions: (a) $\tilde{\mathcal{A}}_{\mu}$ admits a series expansion in the parameter $\frac{q^{2}}{T^{2 / z}}$ i.e.

$$
\tilde{\mathcal{A}}_{\mu}(t, x, r)=\tilde{\mathcal{A}}_{\mu}^{(0)}(t, x, r)+\frac{q^{2}}{T^{2 / z}} \tilde{\mathcal{A}}_{\mu}^{(1)}(t, x, r)+O\left(\frac{q^{4}}{T^{4 / z}}\right)+\cdots \quad \mu \equiv(t, x)
$$

and also, (b) $\left|\partial_{t} \tilde{\mathcal{A}}_{x}\right| \ll\left|\partial_{x} \tilde{\mathcal{A}}_{t}\right|$. The regime of validity of this analysis is

$$
e^{-\frac{T^{2 / z}}{q^{2}}} \ll \frac{\frac{1}{r_{0}}-r_{h}}{\frac{1}{r_{0}}} \ll \frac{q^{2}}{T^{2 / z}} \ll 1 .
$$

The field strength $\tilde{\mathcal{F}}_{t x}$ follows a wave equation: we then choose the ingoing solution. Equivalently, this condition is obtained by demanding that the function $H \equiv r^{\#} f H_{x y}^{\prime}$ depends only on the "infalling" coordinate $v \sim t+\log \left(1 / r_{0}-r\right)$, which gives

$$
\tilde{\mathcal{F}}_{t x}+\left(d_{i}+z-\theta\right) r_{0}^{z}\left(\frac{1}{r_{0}}-r\right) \tilde{\mathcal{F}}_{r x}=0
$$

Alongwith a series of approximations and simplications, we eventually obtain Fick's Law $\tilde{j}^{x}=-\mathcal{D} \partial_{x} \tilde{j}^{t}$ for the variables (C.1) on the stretched horizon.

For $z<d_{i}+2-\theta$, the leading order solution for the gauge field $\tilde{\mathcal{A}}_{t}$ and the shear diffusion constant $\mathcal{D}$ are

$$
\tilde{\mathcal{A}}_{t}^{(0)}=\frac{C e^{-\Gamma t+i q x}}{d_{i}+2-z-\theta} r^{d_{i}+2-z-\theta}, \quad \mathcal{D}=\frac{r_{0}^{z-2}}{d_{i}+2-z-\theta} .
$$

The expression for $\tilde{\mathcal{A}}_{x}^{(0)}$ can be found from $\tilde{\mathcal{A}}_{t}^{(0)}$. Using the above expression for $\mathcal{D}$ along with $(2.2)$ leads to $(2.32)$. In $[25,27]$, we then conjectured $(2.33)$ by examining various special cases: (i) For pure AdS when $z=1, \theta=0$, we obtain $\mathcal{D}=\frac{1}{4 \pi T}$ which along with the thermodynamic relation $\frac{\eta}{s}=\mathcal{D} T$ leads to $\frac{\eta}{s}=\frac{1}{4 \pi}$; (ii) From (2.32) it follows for any hyperscaling violating theory with $z=1$, the $\theta$-dependent prefactors cancel precisely giving 
$\frac{\eta}{s}=\frac{1}{4 \pi}$. This is vindicated for $z=1, \theta \neq 0$ hvLif theories arising from the reduction of non-conformal $D p$-branes on the sphere $S^{8-p}$ [8]: it is well-known that the latter satisfy the viscosity bound and we expect that long-wavelength physics is unaffected by the sphere reduction; (iii) For theories with exact Lifshitz scaling symmetry i.e. $z \neq 0$ and $\theta=0$, the diffusion equation implies the scaling dimension $\operatorname{dim}[\mathcal{D}] \sim z-2$, where $\left[x_{i}\right]=-1$ and $[t]=-z$. Earlier investigations on Lifshitz hydrodynamics $[17,21,22]$ argued that $\frac{\eta}{s}$ exhibits universal viscosity behaviour. This again vindicates the proposed relation (2.33) for consistency. This leads us to the universal viscosity bound (2.33) for $z<d_{i}+2-\theta$, the sector continuously connected to AdS.

When $z=d_{i}+2-\theta$, the leading solution as well as the shear diffusion constant scale logarithmically,

$$
\tilde{\mathcal{A}}_{t}^{(0)}=C e^{-\Gamma t+i q x} \log \frac{r}{r_{c}}, \quad \mathcal{D}=r_{0}^{z-2} \log \frac{1}{r_{0} r_{c}} .
$$

This is reflected in the quasinormal modes through (2.34), (2.35). It is likely that this leading shear diffusion constant undergoes some renormalization/resummation. We note also that $\frac{\eta}{s}$ continues to exhibit universal viscosity behaviour in the Kubo limit as we have seen in section 3.1: perhaps some aspects of hydrodynamics are novel in this case.

For $z>d_{i}+2-\theta$, the series solution breaks down in the near horizon region: the boundary conditions are not satisfied suggesting non-universal UV physics dominates, and we obtain no insight on the shear diffusion constant.

Open Access. This article is distributed under the terms of the Creative Commons Attribution License (CC-BY 4.0), which permits any use, distribution and reproduction in any medium, provided the original author(s) and source are credited.

\section{References}

[1] J.M. Maldacena, The large- $N$ limit of superconformal field theories and supergravity, Int. J. Theor. Phys. 38 (1999) 1113 [hep-th/9711200] [INSPIRE].

[2] S.S. Gubser, I.R. Klebanov and A.M. Polyakov, Gauge theory correlators from noncritical string theory, Phys. Lett. B 428 (1998) 105 [hep-th/9802109] [INSPIRE].

[3] E. Witten, Anti-de Sitter space and holography, Adv. Theor. Math. Phys. 2 (1998) 253 [hep-th/9802150] [INSPIRE].

[4] O. Aharony, S.S. Gubser, J.M. Maldacena, H. Ooguri and Y. Oz, Large-N field theories, string theory and gravity, Phys. Rept. 323 (2000) 183 [hep-th/9905111] [INSPIRE].

[5] S.A. Hartnoll, A. Lucas and S. Sachdev, Holographic quantum matter, arXiv:1612.07324 [INSPIRE].

[6] N. Ogawa, T. Takayanagi and T. Ugajin, Holographic Fermi Surfaces and Entanglement Entropy, JHEP 01 (2012) 125 [arXiv:1111.1023] [INSPIRE].

[7] L. Huijse, S. Sachdev and B. Swingle, Hidden Fermi surfaces in compressible states of gauge-gravity duality, Phys. Rev. B 85 (2012) 035121 [arXiv:1112.0573] [INSPIRE].

[8] X. Dong, S. Harrison, S. Kachru, G. Torroba and H. Wang, Aspects of holography for theories with hyperscaling violation, JHEP 06 (2012) 041 [arXiv: 1201.1905] [INSPIRE]. 
[9] K. Narayan, On Lifshitz scaling and hyperscaling violation in string theory, Phys. Rev. D 85 (2012) 106006 [arXiv:1202.5935] [INSPIRE].

[10] H. Singh, Lifshitz/Schródinger Dp-branes and dynamical exponents, JHEP 07 (2012) 082 [arXiv: 1202.6533] [INSPIRE].

[11] K. Narayan, T. Takayanagi and S.P. Trivedi, AdS plane waves and entanglement entropy, JHEP 04 (2013) 051 [arXiv: 1212.4328] [INSPIRE].

[12] K. Narayan, Lightlike limit of entanglement entropy, Phys. Rev. D 91 (2015) 086010 [arXiv: 1408.7021] [INSPIRE].

[13] K. Narayan, Non-conformal brane plane waves and entanglement entropy, Phys. Lett. B 726 (2013) 370 [arXiv: 1304.6697] [INSPIRE].

[14] P. Kovtun, D.T. Son and A.O. Starinets, Viscosity in strongly interacting quantum field theories from black hole physics, Phys. Rev. Lett. 94 (2005) 111601 [hep-th/0405231] [INSPIRE].

[15] D.-W. Pang, Conductivity and Diffusion Constant in Lifshitz Backgrounds, JHEP 01 (2010) 120 [arXiv:0912.2403] [INSPIRE].

[16] S. Cremonini and P. Szepietowski, Generating Temperature Flow for $\eta / s$ with Higher Derivatives: From Lifshitz to AdS, JHEP 02 (2012) 038 [arXiv:1111.5623] [INSPIRE].

[17] C. Hoyos, B.S. Kim and Y. Oz, Lifshitz Field Theories at Non-Zero Temperature, Hydrodynamics and Gravity, JHEP 03 (2014) 029 [arXiv: 1309.6794] [INSPIRE].

[18] J. Sadeghi and A. Asadi, Hydrodynamics in a black brane with hyperscaling violation metric background, Can. J. Phys. 92 (2014) 1570 [arXiv:1404.5282] [INSPIRE].

[19] D. Roychowdhury, Holographic charge diffusion in non relativistic branes, Phys. Lett. B $\mathbf{7 4 4}$ (2015) 109 [arXiv: 1412.0911] [INSPIRE].

[20] D. Roychowdhury, Hydrodynamics from scalar black branes, JHEP 04 (2015) 162 [arXiv: 1502.04345] [INSPIRE].

[21] E. Kiritsis and Y. Matsuo, Charge-hyperscaling violating Lifshitz hydrodynamics from black-holes, JHEP 12 (2015) 076 [arXiv: 1508.02494] [INSPIRE].

[22] E. Kiritsis and Y. Matsuo, Hyperscaling-violating Lifshitz hydrodynamics from black-holes: Part II, JHEP 03 (2017) 041 [arXiv: 1611.04773] [INSPIRE].

[23] X.-M. Kuang, X.-M. Kuang, J.-P. Wu and J.-P. Wu, Analytical shear viscosity in hyperscaling violating black brane, Phys. Lett. B 773 (2017) 422 [arXiv:1511.03008] [inSPIRE].

[24] M. Blake, Universal Charge Diffusion and the Butterfly Effect in Holographic Theories, Phys. Rev. Lett. 117 (2016) 091601 [arXiv: 1603.08510] [INSPIRE].

[25] K.S. Kolekar, D. Mukherjee and K. Narayan, Hyperscaling violation and the shear diffusion constant, Phys. Lett. B 760 (2016) 86 [arXiv:1604.05092] [INSPIRE].

[26] A. Eberlein, A.A. Patel and S. Sachdev, Shear viscosity at the Ising-nematic quantum critical point in two dimensional metals, Phys. Rev. B 95 (2017) 075127 [arXiv:1607.03894] [INSPIRE].

[27] K.S. Kolekar, D. Mukherjee and K. Narayan, Notes on hyperscaling violating Lifshitz and shear diffusion, Phys. Rev. D 96 (2017) 026003 [arXiv:1612.05950] [INSPIRE]. 
[28] P.K. Kovtun and A.O. Starinets, Quasinormal modes and holography, Phys. Rev. D 72 (2005) 086009 [hep-th/0506184] [INSPIRE].

[29] A.O. Starinets, Quasinormal spectrum and the black hole membrane paradigm, Phys. Lett. B 670 (2009) 442 [arXiv:0806.3797] [INSPIRE].

[30] A. Núñez and A.O. Starinets, AdS/CFT correspondence, quasinormal modes and thermal correlators in $N=4$ SYM, Phys. Rev. D 67 (2003) 124013 [hep-th/0302026] [INSPIRE].

[31] E. Berti, V. Cardoso and A.O. Starinets, Quasinormal modes of black holes and black branes, Class. Quant. Grav. 26 (2009) 163001 [arXiv:0905. 2975] [INSPIRE].

[32] P. Kovtun, D.T. Son and A.O. Starinets, Holography and hydrodynamics: Diffusion on stretched horizons, JHEP 10 (2003) 064 [hep-th/0309213] [INSPIRE].

[33] D.T. Son and A.O. Starinets, Minkowski space correlators in AdS/CFT correspondence: Recipe and applications, JHEP 09 (2002) 042 [hep-th/0205051] [INSPIRE].

[34] G. Policastro, D.T. Son and A.O. Starinets, From AdS/CFT correspondence to hydrodynamics, JHEP 09 (2002) 043 [hep-th/0205052] [INSPIRE].

[35] I.R. Klebanov and E. Witten, AdS/CFT correspondence and symmetry breaking, Nucl. Phys. B 556 (1999) 89 [hep-th/9905104] [INSPIRE].

[36] S. Bhattacharyya, V.E. Hubeny, S. Minwalla and M. Rangamani, Nonlinear Fluid Dynamics from Gravity, JHEP 02 (2008) 045 [arXiv:0712.2456] [INSPIRE]. 